\title{
IAMG Andrei Borisovich Vistelius Research Award of the Year 2021: Vanessa A. Godoy
}

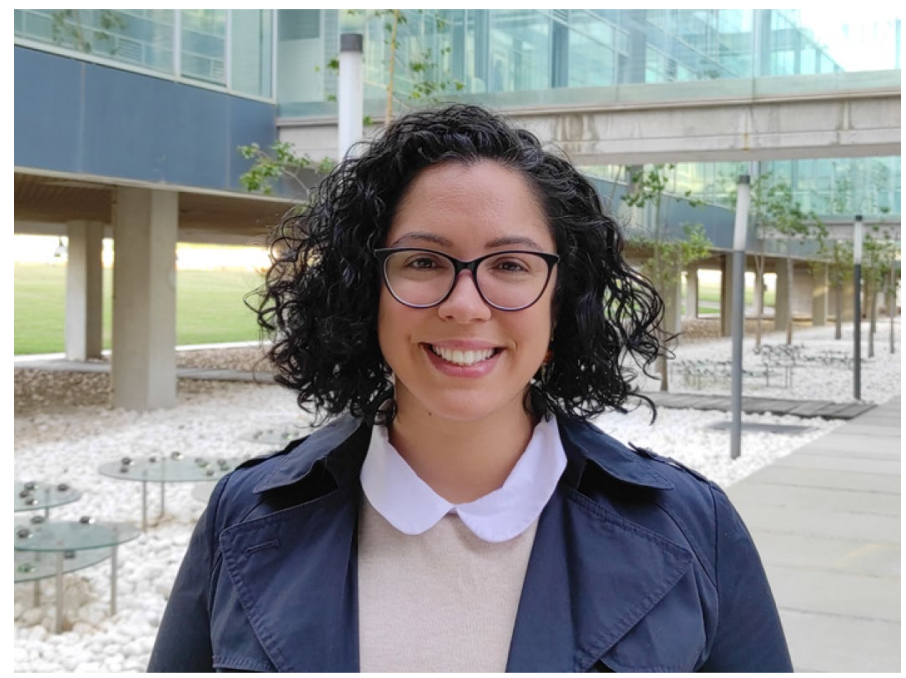

Vanessa A. Godoy in front of the Research Park at the Universitat Politècnica de València

On January 15th, 2014, I received an email from a Ph.D. student at the Universidade de São Paulo in Brazil, whom I did not know, nor had I contacted in the past. I did not imagine that it would be the beginning of a long and fruitful academic relationship that brought me to Brazil on three occasions for extended periods, and that ended with Vanessa as a postdoctoral researcher in my group at the Institute for Water and Environmental Engineering in Valencia and she receiving the most prestigious award for young scientists working in mathematical geosciences bestowed by the International Association for Mathematical Geosciences. Congratulations, Vanessa! 
Vanessa is a remarkable woman who has fought through the many obstacles facing women in Brazil, to obtain a double Ph.D. from the Universidade de São Paulo and Universitat Politècnica de València and then to win one of the highly competitive fellowships for women from developing and emerging economies granted by the Schlumberger Foundation Faculty for the Future - a fellowship that was recently renewed for a second year.

She graduated in 2018 and has published four papers in journals positioned in the first quartile of the Journal Citation Reports in their fields, plus dozens of other papers in lesser-impact journals. Currently, we are working on three more papers that are under review or ready to be submitted. The papers related to her Ph.D. concern the analysis of heterogeneity of different parameters that control water flow and mass transport in tropical soils, with, for the first time, a comparison between the long-standing theoretical results and laboratory analyses carried out at different scales. The results are very interesting, for two reasons: the first one is that tropical soils display characteristics different from regular soils, and the second is that the laboratory analyses have corroborated some theoretical upscaling results.

Given the difficulty in getting funding from the Brazilian government, which has basically shut down research financing in the past few years, Vanessa had to seek funding elsewhere, which allowed her to travel to Spain to complete her double Ph.D. and to attend several international conferences.

We are in front of a young, strong-minded, and promising researcher whose contributions are outstanding. For this reason, I wholeheartedly nominated her for the prestigious Andrei Borisovich Vistelius Research Award that she eventually won.

J. Jaime Gómez-Hernández Universitat Politècnica de València

Spain 